\title{
Application of the state observer in optimal control system
}

\author{
Hao $\mathrm{Xu}^{1, \mathrm{a}, \dagger}$ and Ze Dong ${ }^{2, \mathrm{~b}}$ \\ ${ }^{1}$ Hebei Engineering Research Center of Simulation \& Optimized Control for \\ Power Generation \\ , Baoding, China, 071003 \\ ${ }^{a}$ xu20hao10@163.com, ${ }^{b}$ dongze33@126.com \\ ${ }^{\dagger}$ Corresponding author
}

\begin{abstract}
In today's industry, optimal control system which has reliable, stable performance and good man-machine interface is widely used. And state observer can solve the problem of the state reconstruction of the controlled system and create the condition for realizing state feedback. In this paper, the application of the relevant theoretical knowledge of state observer and state feedback pole assignment in the optimal control system will be studied and the control result is very good.
\end{abstract}

Keywords: Optimal Control System, State Observer, State Feedback, Application.

\section{Introduction}

Optimal control system which is based on DCS system, adopts the design thought of control dispersion and management focus. Its control effect is reliable and stable. Now it has been extremely widespread applied in electric power, metallurgy, petrochemical, and all walks of life.

With the development of the industry, there are more and more high order time, big delay controlled plant appeared, the traditional method has been difficult to obtain better control effect. And the state observer theory proposed by Luenberger solves the problem of the controlled system state reconstruction. When the system is completely considerable, the state feedback control law has become a reality, the physical implementation of state observer become possible.

So, the theory of pole assignment and state observer in modern control theory is applied to the optimal control system with high reliability. In combination with other control methods, it can achieve better control effect. 


\section{The Realization of State Observer}

\subsection{Introduce state observer theory.}

In the modern control theory, the optimal control system and decoupled system are dependent on the state feedback. However, the system states are not available with physical method, some even can't measure. Then it puts forward the concept of state observer due to the difficult of physical implementation of state feedback.

The essence of state reconstruction is that constructing a linear time-invariant system $\Sigma$ which is similar to original linear time-invariant system ${ }^{\Sigma_{0}}$ [1]. Take the system output and control output in system $\Sigma_{0}$ as the input of system $\Sigma$, and make the state of $\Sigma$ and $\Sigma_{0}$ gradually converged. In other words, two state is distinct originally, but they are gradually tend to be consistent with the increase of time, as in

$$
\lim _{t \rightarrow \infty}|\hat{x}-x|=0
$$

The state $x$ of linear time-invariant system $\Sigma_{0}(A, B, C)$ cannot be directly measured. The state $\hat{x}$ will be close to the state $x$, when system $\hat{\Sigma}$ takes the system output and control output in system ${ }^{\Sigma_{0}}$ as its input. So, $\hat{\Sigma}_{\text {is }}$ the state observer of system ${ }^{\Sigma_{0}}$.

According to the definition above, the principle of constructing a state observer is:

(1)The state observer $\hat{\Sigma}$ should take the system output and control output in system $\Sigma_{0}$ as the input.

(2)To meet the formula $\lim _{t \rightarrow \infty}|\hat{x}-x|=0$, system ${ }^{\Sigma_{0}}$ should be observable or the unobservable part is asymptotic stable.

(3)The output $x$ of $\Sigma$ hould tend to $x$ fast enough.

\subsection{Transfer function transform into state space expression.}

The transfer function of controlled plant is:

$$
G(s)=\frac{K}{(T s+1)^{n}}
$$


According to the principle of series, disintegrating $\mathrm{n}$ order transfer function into the product of some first-order transfer function [2]. Then link them together and choose the output of every integrator as state variable. Eventually, the corresponding state-space equation is:

$$
\left\{\begin{array}{c}
\dot{x}=\left[\begin{array}{cccc}
-\frac{1}{T} & 0 & \cdots & 0 \\
\frac{1}{T} & -\frac{1}{T} & \cdots & 0 \\
\vdots & \vdots & \vdots & \vdots \\
0 & \cdots & \frac{1}{T} & -\frac{1}{T}
\end{array}\right]\left[\begin{array}{c}
x_{1} \\
x_{2} \\
\vdots \\
x_{n}
\end{array}\right]+\left[\begin{array}{c}
\frac{K}{T} \\
0 \\
\vdots \\
0
\end{array}\right] u \\
y=\left[\begin{array}{llll}
0 & 0 & \cdots & 1
\end{array}\right]\left[\begin{array}{c}
x_{1} \\
x_{2} \\
\vdots \\
x_{n}
\end{array}\right]
\end{array}\right.
$$

In this way, the transformation between the transfer function and the state space expression is realized.

\subsection{The discrete processing of state observer equation.}

The system is:

$$
\left\{\begin{array}{c}
\dot{x}=A x+B u \\
y=C x
\end{array}\right.
$$

If the system is completely observable, the corresponding observable matrix is:

$$
V_{0}=\left[\begin{array}{c}
C \\
C A \\
\vdots \\
C A^{n-1}
\end{array}\right]
$$

And the feedback matrix of state observer is: 


$$
L=f_{0}^{*}(A) V_{0}^{-1}\left[\begin{array}{c}
0 \\
0 \\
\vdots \\
1
\end{array}\right]
$$

So, the state equation of state observer is:

$$
\hat{x}=(A-L C) \hat{x}+B u+L y
$$

$\hat{x}$ The discrete processing of state equation is necessary if we want to edit $\underset{\text { program in }}{x_{(k+1)}-x_{(k)}}$ optimal control system [3]. That is to say, replacing $x$ with

$\Delta t \quad$. The final equation is obtained as in:

$$
\hat{x}_{(k+1)}=\hat{x}_{(k)}+\Delta t\left[(A-L C) x_{(k)}+B u+L y\right]
$$

\subsection{Frame of state observer in optimized control system.}

In conclusion, the state observer is realizable, which takes the system output and control output as its input. The output of controlled plant is state.

The figure in optimal control system is shown in Fig.1 and Fig.2:

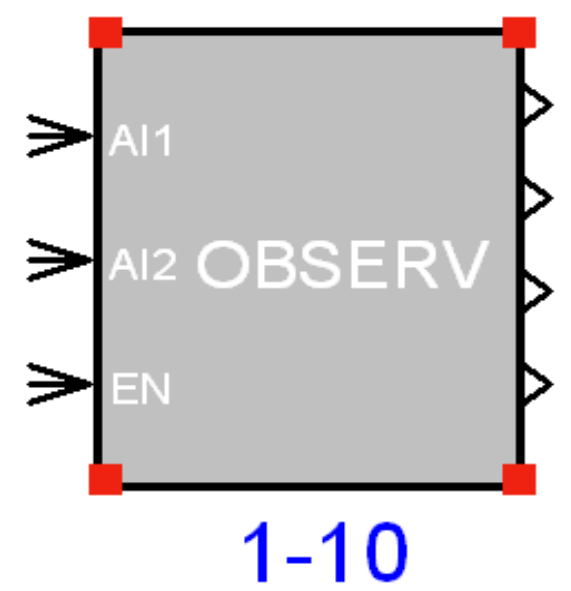

Fig.1 The module of state observer. 


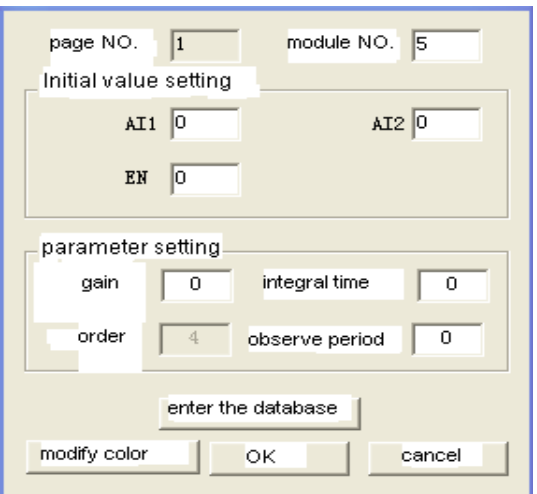

Fig.2 Parameter setting box

In the figure above, the Fig. 1 is module of state observe and the Fig. 2 is parameter Settings bar. Proportional gain, integral time, the order and observed period can be inputted in parameter Settings bar.

Therefor, the control logic based on state observer can be constructed.

\section{The Principle of Select State Feedback Pole}

Comparing with traditional PID controller, using state feedback control can be easily configured closed-loop pole position. Direct benefit is transforming the features of the system, improving the control precision. There is no doubt that it is a good control scheme for controlled plant of big inertia and large delay [4].

There is not the only and universal optimal law on the choice of the eigen root of the matrix in dynamic feedback system, because it is a complicated system itself and properties of the system are mutually restricted. So it is impossible that all systems and all design requirements have a single and universal optimal selection law of pole, and that design the best pole position without debugging. State feedback matrix depends on the expected pole position and the principle of making the error due to interference quickly reduced to zero. And closed-loop poles determine the speed of response. The location of expected closed-loop poles reflects the system response speed and stability [5]. And also take into account the reason of actual system controller output is limited.

Based on the above considerations, there are some basic understandings about pole position effecting the comprehensive performance of the system, which can be used to guide the choice of the pole.

(1)The bigger the absolute value of negative real part of the pole, the faster the system achieve steady response.

(2)According to the root locus angle, the farther from the poles of a feedback control system to the poles of open-loop transfer function, the bigger the 
feedback control gain, and the more unstable the feedback system. And, being limited by the linear region of the actuator, the control effect can not reach ideal level. In addition, Actuator movement too often will have a negative impact on the equipment. Therefore, it is not right that the pole assignment of the controlled plant from the imaginary axis as far as possible.

This is the restriction of first understanding. The above emphasizes the performance, this shows that the high performance system often have a high sensitivity [6].

(3)Repeated eigenvalues makes the transient response of the system not smooth which makes the feedback matrix parameters sensitive. So we should try to avoid repeated eigenvalues, even eigenvalues of closely together. There will always be mutative parameters in system model of the actual operation, and the close eigenvalues will make the system unstable.

Above all, the selection of state feedback pole is not easy, and there is not fixed laws and formulas. Getting the right pole is a trial-and-error process and needs pay attention to the actual control effect. Meanwhile, if the controlled system can control and can be observed, the assignment of state feedback pole and the design of observer can be processed on one's own when structure state feedback system with state observer.

\section{Example of a Control Application}

Select a controlled plant with big order time and inertia as follows [7]:

$$
G(s)=\frac{0.9}{(19 s+1)^{4}}
$$

Input the corresponding parameters to the input box as shown in Fig.3:

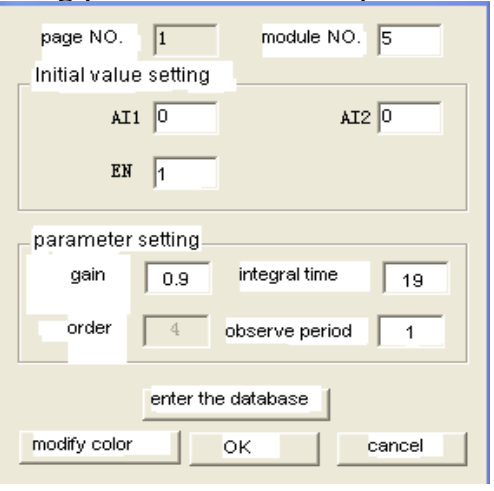

Fig.3 Parameter setting box 
In the actual control, because of the single state observer control has steady-state error, add to the PID controller in the logical system. And then structure state observer and PID controller combined control system. The logic diagram is shown in Fig.4 and the logic diagram in optimal control system is shown in Fig.5.

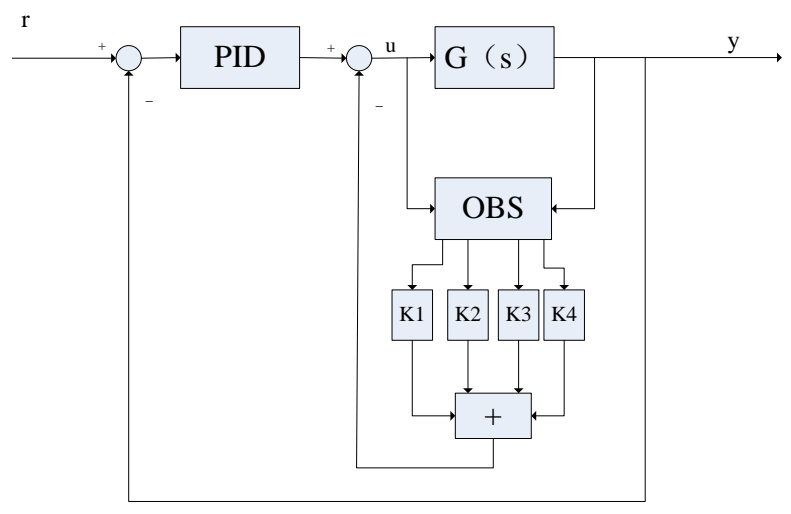

Fig.4 logic diagram.

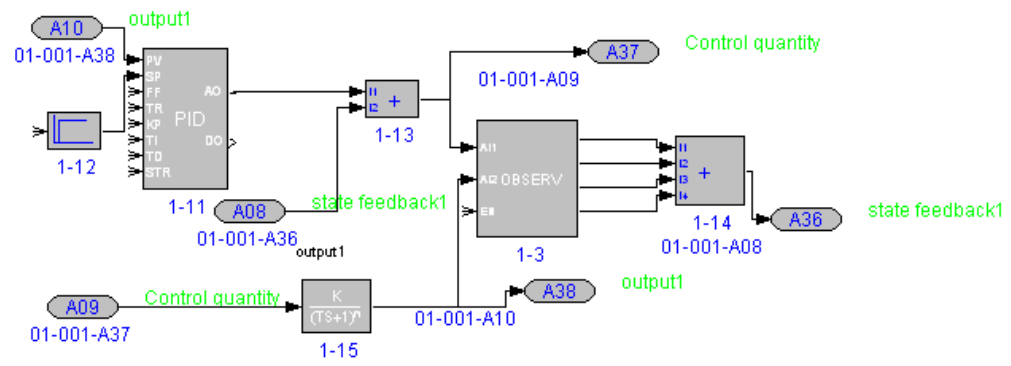

Fig.5 logic diagram.

Based on the system dynamic performance requirements, choose a set of suitable control pole as, $\mathrm{p}=\left[\begin{array}{l}-0.09+\mathrm{j} * 0.03-0.09-\mathrm{j} * 0.03-0.1-0.12] \\ \text {. The }\end{array}\right.$ corresponding state feedback matrix is, $\mathrm{K}=$ [4 5.6413 .8261 .061$]$.

After system begin operation, get the system output curve is shown in Fig.6. 


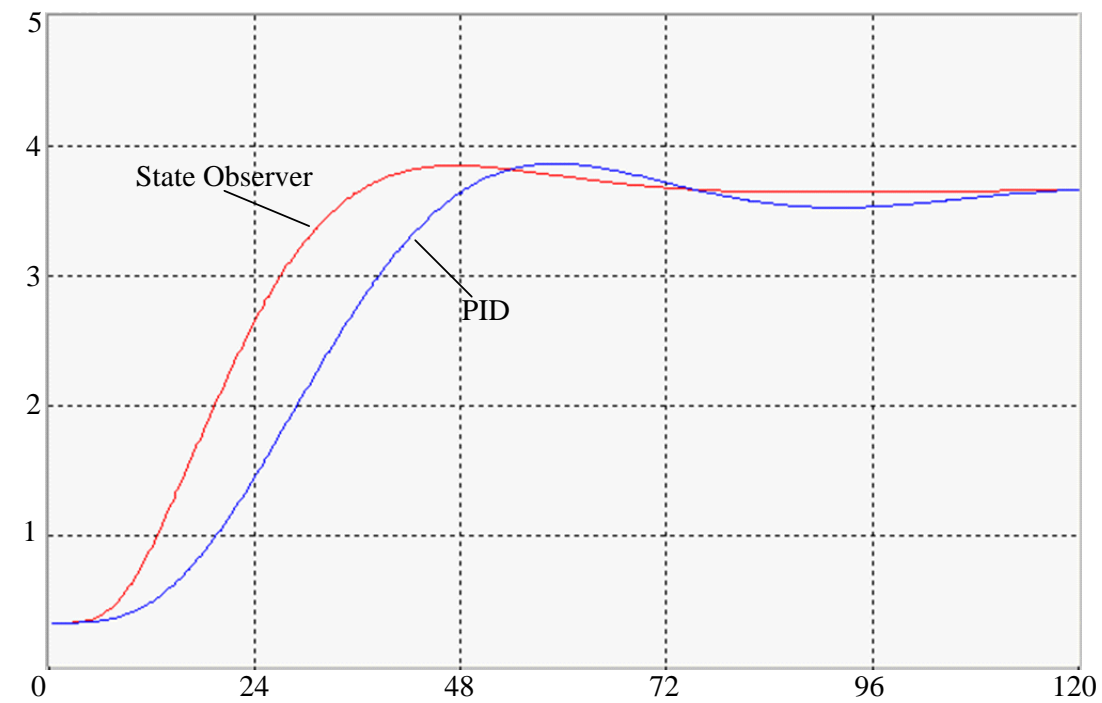

Fig.6 System output curve

The red line is state observer combined with PID controller output curve and the blue line is output curve of single PID controller.

Obviously, the first kind of control strategy is better than the single PID control on the dynamic performance and stability.

\section{Summary}

After analyzing the possibility that a state observer is realized in the optimized control system and adding state observer to the system, we can get the conclusion that the control strategy based on state observer is more stable and speedy, which has very good application value.

\section{Acknowledgement}

I am very grateful to my tutors Ze Dong and Dong-feng Wang who give me a lot of useful advice and help on my thesis. Special thanks should go to my friends and my classmates whose selfless pay and close cooperation let me gain a lot. Finally, I would like to thank my parents and family members for their loving considerations and great confidence in me all through these years. 


\section{References}

[1] Keming Xie. Foundation of modern control theory. Beijing University of Technology press, 2006,02:207-219. In Chinese.

[2] Bao-Zhu Guo,Zhi-Liang Zhao. Extended State Observer for Nonlinear Systems with Uncertainty[J]. IFAC Proceedings Volumes,2011,441:. In Chinese.

[3] K Z. Xu, Z. Wang and Y. Wang, "Optimal control of nonlinear system based on linear extended state observer," Control Conference (CCC), 2011 30th Chinese, Yantai, 2011, pp. 97-101. In Chinese.

[4] Xiu-Chun Luan, Shi-Yong Li and Li-Ling Li, "Adaptive control for the superheated steam temperature based on multi-model state observer," Machine Learning and Cybernetics, 2004. Proceedings of 2004 International Conference on, 2004, pp. 978-982 vol.2. . In Chinese.

[5] Yanjun Zhang,Jun Zhang,Lu Wang,Jianbo Su. Composite disturbance rejection control based on generalized extended state observer[J]. ISA Transactions, 2016.: In Chinese.

[6] Victor A. Unkin,Svetlana A. Krasnova,Anton V. Utkin. State Observers in Control Systems of Induction Motor Drives[J]. IFAC Proceedings Volumes,2013,469:.

[7] Yinsong Wang, Zunji Li, Yuping Liu, Zhigang Zhang, Jinzhu Cai. Design and application of the state observer main steam temperature control system [J]. Journal of North China Electric Power Institute, 1994,02:104-108. In Chinese. 